\title{
New Expression for Collisionless Magnetic Reconnection Rate
}

\author{
Alex Klimas ${ }^{1}$ \\ ${ }^{1}$ GPHI at University of Maryland, Baltimore County, Baltimore, Maryland 21228, USA
}

\begin{abstract}
For 2D, symmetric, anti-parallel, collisionless magnetic reconnection, a new expression for the reconnection rate in the electron diffusion region is introduced. It is shown that this expression can be derived in just a few simple steps from a physically intuitive starting point; the derivation is given in its entirety and the validity of each step is confirmed. The predictions of this expression are compared to the results of several long-duration, open-boundary PIC reconnection simulations to demonstrate excellent agreement.
\end{abstract}

\section{Introduction}

At Earth's day-side magnetopause and in its magnetotail, magnetic reconnection plays a key role in organizing the overall structure of the magnetosphere ${ }^{1,2}$. At both positions, collisionless reconnection is generally accepted as the appropriate model ${ }^{3}$. In the absence of effective collisions, it has been proposed that off-diagonal electron pressure tensor elements and electron inertia support the reconnection electric field in the electron diffusion region (EDR), thereby enabling reconnection ${ }^{4-6}$. Simulation studies are available that support this explanation ${ }^{7-11}$ and models of the electron motion in the EDR have been constructed that explain the presence of the off-diagonal electron pressure tensor elements and electron inertia ${ }^{12,13}$. Under the assumption that the off-diagonal tensor elements and inertia contribution are weak compared to the diagonal pressure tensor elements, linear approximations that estimate the strength of the reconnection electric field have been developed ${ }^{14-16}$.

In this paper, an alternate approach to an expression for the collisionless reconnection rate is presented. This development is limited to $2 \mathrm{D}$, symmetric, anti-parallel reconnection at present. The strength of the electron current sheet at a reconnection site is estimated on the assumption that the average electron velocity there is a gyration velocity about the normal magnetic field components in the outflow jets. As will be shown below, this assumption leads to a notably accurate estimate of the current sheet strength. With this starting point, a few simple steps lead to a new expression for the reconnection rate. Assuming that the ion contribution to the total current sheet strength can be ignored and that the out-of-plane reconnection electric field strength is uniform over the EDR, an expression for the reconnection rate in the EDR follows

$$
\frac{v_{\text {in }}}{v_{\text {eAin }}}=4 \frac{\alpha}{\hat{L} \hat{d}}
$$


in which $v_{i n}$ is the electron inflow speed at the inflow boundary to the EDR, $v_{\text {eAin }}$ is the electron Alfvén speed at the same position, $\hat{L}$ is the EDR length on the outflow axis measured in units of the local electron inertial length, $\hat{d}$ is the width of the EDR or of the central electron current sheet on the inflow axis, again measured in units of the local electron inertial length, and $\alpha=0.45$ is a dimensionless constant defined below.

A long duration $\left(500 \omega_{c i}^{-1}\right)$, open-boundary, driven PIC reconnection simulation has been used to guide the derivation of (1). This derivation and the supporting simulation results are presented below. The degree to which the left and right sides of (1) are in agreement demonstrates the accuracy of this reconnection rate expression. An additional group of related driven and undriven simulations has been used to more generally demonstrate the accuracy of (1). This step has shown that $\alpha$ is independent of driving rate and mass ratio, thus suggesting the possibility of a universal dimensionless constant.

The unexpected $(L d)^{-1}$ dependence of the reconnection electric field that follows from (1) is in apparent contradiction with the behavior $E_{\text {rec }} \sim d / L$ that has been shown ${ }^{17,18}$ to follow from fundamental MHD conservation laws. A new expression for the EDR width $\hat{d}$ is introduced that removes this apparent contradiction. This expression provides the link between the conservation law approach to the reconnection rate and the "electron turning" approach discussed in this paper. The two approaches are found complementary and compatible.

\section{Simulation Setup}

A 21/2-dimensional electromagnetic PIC code described in Hesse et al. ${ }^{15}$ has been modified to incorporate open inflow and outflow boundary conditions ${ }^{19-21}$. Densities and fluxes are accumulated on the grid using a rectangular particle shape function. Ghost particles required by the zero-gradient boundary condition on the particle distributions contribute to the densities and fluxes at the open boundaries at each time step. The electromagnetic fields are integrated implicitly to avoid the Courant constraint on the propagation of light waves ${ }^{15}$. Lengths are normalized with respect to the ion inertial length $d_{i}=c / \omega_{p i}=c\left(e^{2} n_{0} / \varepsilon_{0} m_{i}\right)^{-1 / 2}$ using the initial current sheet density $n_{0}$, time is normalized to the inverse ion cyclotron frequency $\omega_{c i}=e B_{0} / m_{i}$ using the asymptotic magnetic field strength $B_{0}$, and velocities are normalized to the Alfvén speed $V_{A 0}$ computed using $n_{0}$ and $B_{0}$. An $(x, y, z)$ coordinate system is used with the outflow and inflow directions on the $x$ - and $z$-axes respectively and with out-of-plane directions on the $y$-axis. The initial equilibrium configuration is a Harris sheet

$$
B_{x}=\tanh (z / \lambda)
$$

with an additional perturbation given by

$$
B_{x p}=-\frac{a_{0} \pi}{L_{z}} \sin \left(\pi x / L_{x}\right) \sin \left(\pi z / L_{z}\right)
$$


and

$$
B_{z p}=-\frac{a_{0} \pi}{L_{x}} \cos \left(\pi x / L_{x}\right) \cos \left(\pi z / L_{z}\right)
$$

with $\lambda=0.5 d_{i}$ and $a_{0}=0.25$, leading to a $0.5 \%$ perturbation field. No guide field is included.

The simulations discussed below were initialized with approximately $(2-4) \times 10^{8}$ particles on a $1600 \times 1200$ grid in the $x \times z$ directions. Due to the open boundaries, these numbers vary with increasing simulation time. Four particle species, two of ions and two of electrons were included. The foreground set of ions and electrons was initialized to establish the pressure and current densities required by (2)(4) and the background set was initialized to provide a constant background density $n_{b}=0.2$.

Background and foreground particle temperatures were initialized with $T_{i}=T_{e}=0.25$. Using the asymptotic magnetic field strength to define the electron cyclotron frequency $\omega_{c e}=e B_{0} / m_{e}$ and the current sheet density $n_{0}$ for the electron plasma frequency $\omega_{p e}=\left(e^{2} n_{0} / \varepsilon_{0} m_{e}\right)^{1 / 2}, \omega_{p e} / \omega_{c e}=2$ has been chosen. Further details can be found in Klimas et al. ${ }^{19-21}$.

The results of a group of simulations have been used to derive and test the reconnection rate expression (1). Of these, the primary simulation that will be used to demonstrate the derivation will be denoted "run60". This is a driven simulation with mass ratio $m_{i} / m_{e}=100$ on a domain $L_{x}=80 d_{i}$ by $L_{z}=40 d_{i}$. The remaining simulations were driven by varying rates, or undriven, with mass ratio $m_{i} / m_{e}=25$ on the domain $L_{x}=160 d_{i}$ by $L_{z}=80 d_{i}$. This group will be used to demonstrate the performance of the derived reconnection rate expression as well as to provide some evidence for the possible universality of the parameter $\alpha$.

\section{EDR Definition}

Using the electron momentum moment equation, the EDR is defined as that region surrounding the $x-$ line position in which the out-of-plane non-ideal electric field component

$$
E_{y}^{*}=E_{y}+\left[\mathbf{v}_{e} \times \mathbf{B}\right]_{y}=-\left[\frac{\partial \mathbf{v}_{e}}{\partial t}+\mathbf{v}_{e} \cdot \nabla \mathbf{v}_{e}+\frac{1}{n_{e}} \nabla \cdot \mathbf{P}_{e}\right]_{y}>0
$$

From (1), it can be seen that the dimensions of the EDR play an important role in defining the reconnection rate. These are defined in some detail in this section.

Taken from run60 at approximately the midpoint of the run, panel (a) of Figure 1 shows $E_{y}^{*}(z)$ on a cut through the EDR in the $z$-direction at the position of the $x$-line. The heavy vertical lines show the positions surrounding the x-line at which $E_{y}^{*}=0$. These mark the boundaries of the EDR in the $z$ direction and the distance between them is the width $d$ of the EDR. Panel (b) shows the out-of-plane component of the electron current $j_{e y}(z)$ on the same cut through the EDR. It can be seen that the 
width of the EDR is also the width of the central peak in the electron current sheet. The zeroes in $E_{y}^{*}(z)$ have been used to measure the width $d$ but, as will become evident below, the width of the current sheet peak is of equal importance.
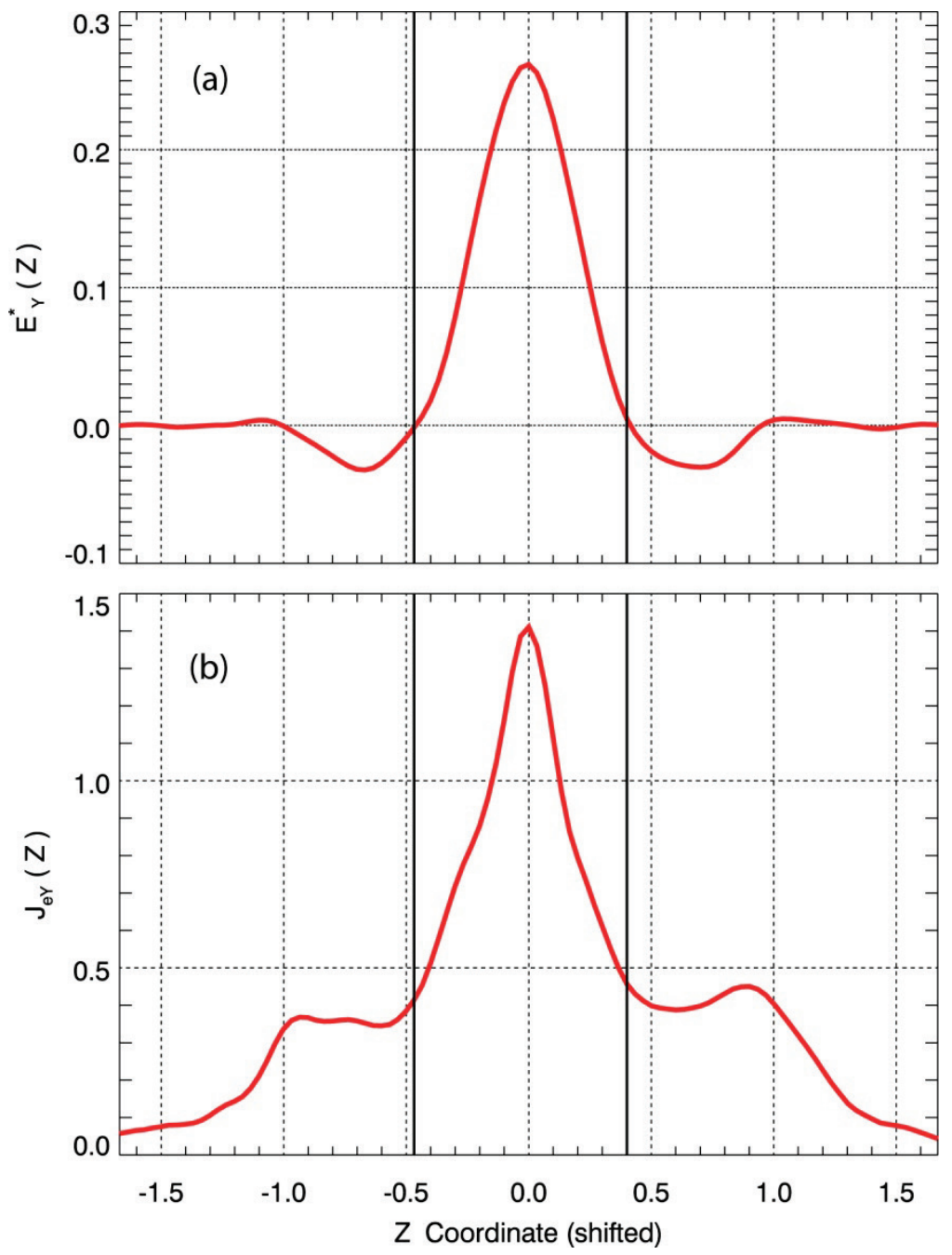

Figure 1: (color online) (a) $E_{y}^{*}(z)$ and (b) $j_{e y}(z)$ both on a cut through the x-line position.

The $z$ coordinate has been shifted so that $z=0$ is at the $x$-line position. Both curves have been time-averaged over a small portion of an ion gyro-period and smoothed with 5-point box-car averaging in $z$.

The length of the EDR can be measured in the same way as the width, by finding the zeroes in $E_{y}^{*}(x)$ while moving away from the $x$-line in the outflow directions; the length $L$ is defined as the distance between the two zeroes. A turning length also can be defined. Moving away from the $x$-line in an outflow direction, a point can be found at which the gyro-radius of a thermal electron in the outflow $B_{z}$ field is equal to the distance from that point to the $x$-line. This point is defined as a turning point and the turning length is the distance between the two turning points. Figure 2 shows the evolution of the EDR length over the course of run60 with 1.15 times the turning length superposed. With this multiplicative 
factor included, the two lengths are essentially identical. Similar results have been found earlier with a multiplicative factor 1.3 for mass ratio 25 simulations ${ }^{20}$. This suggests that the two lengths may converge as the mass ratio is increased. For the analysis discussed below of the mass ratio 100 run60, these two lengths have been considered interchangeable.

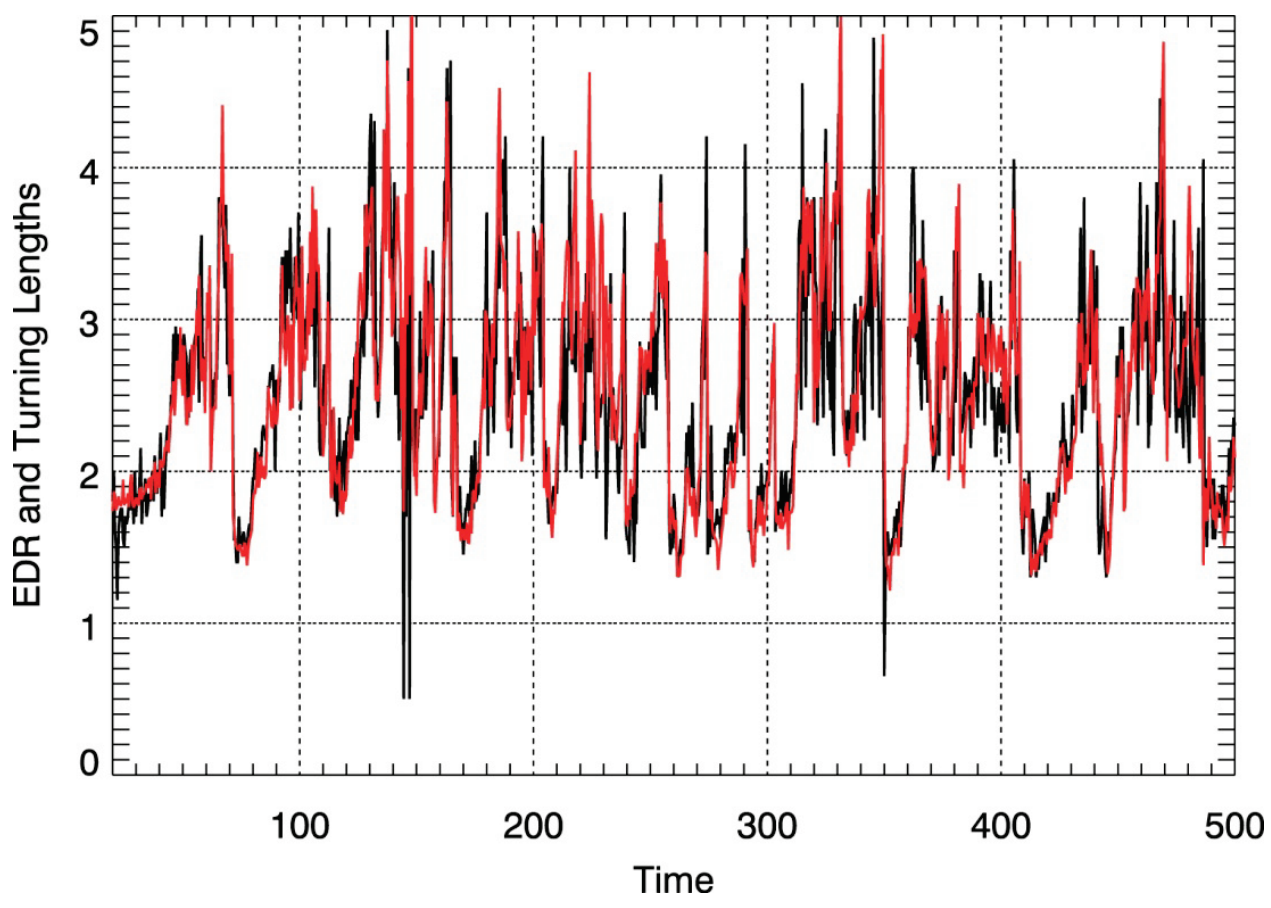

Figure 2: (color online) Black: EDR length as defined through zeroes in $E_{y}^{*}(x)$.

Red (gray): Turning length multiplied by constant factor 1.15 .

\section{Electron Turning}

This first step in obtaining the reconnection rate expression (1) is to estimate the strength of the electron current sheet at the x-line; i.e., the strength of the peak in $j_{e y}$ shown in Figure 1 . Assuming that this group of electrons moves freely in the out-of-plane direction while they are turned out of the current sheet and into the outflow directions through gyration about the outflow $B_{z}$ field, a possible estimate is given by

$$
\bar{j}_{e y}=n_{e_{X L}} \omega_{e c_{T P}} \frac{L}{2}
$$

in which $\bar{j}_{e y}$ is the average of $j_{e y}$ over the width $d, n_{e_{X L}}$ is the electron density at the x-line, and $\omega_{e c_{T P}}$ is the electron cyclotron frequency in the $B_{z}$ field at a turning point (later denoted $B_{\text {out }}$ ). The factor $L / 2$ plays the role of a gyro-radius. 


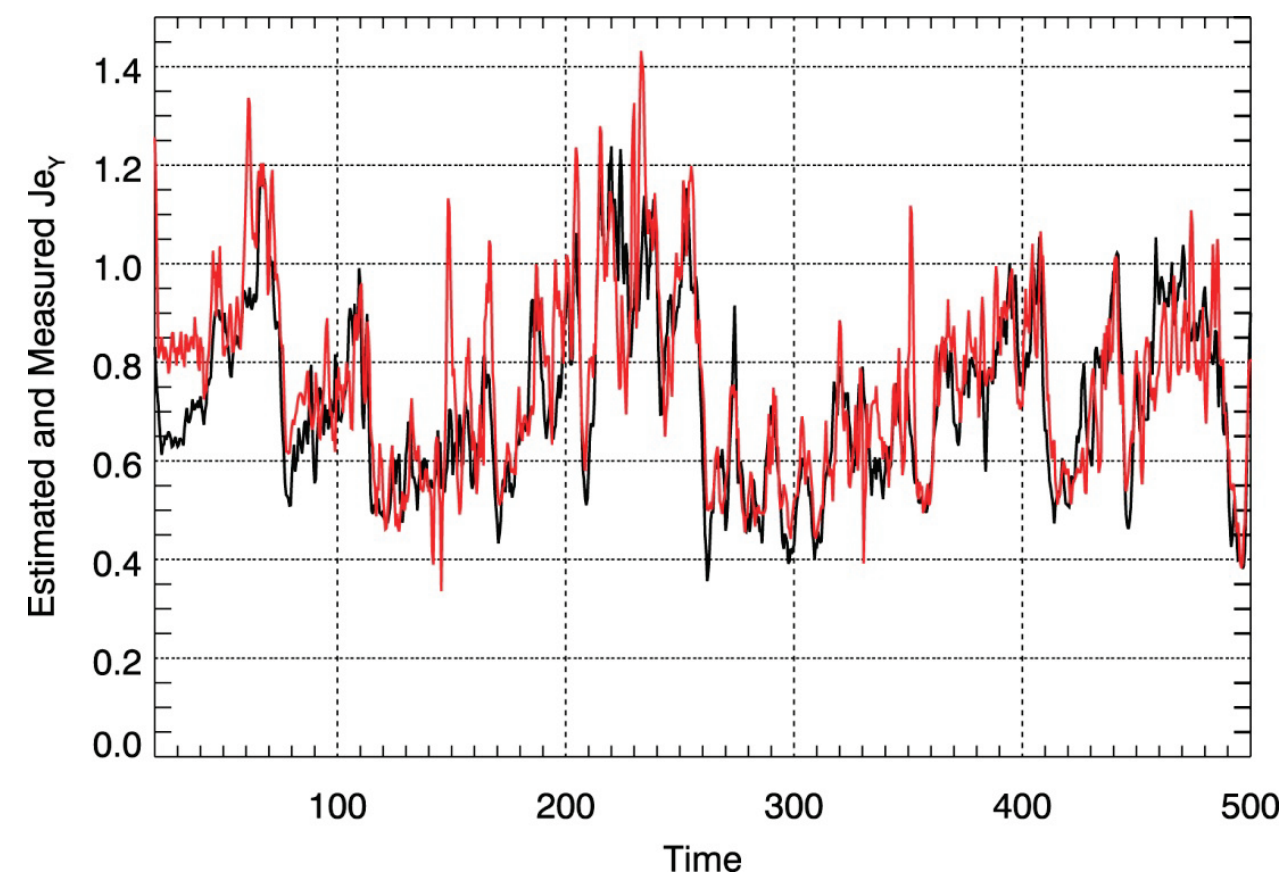

Figure 3: (color online) Black: Measured $\bar{j}_{e y}$. Red (gray): $\bar{j}_{e y}$ predicted by (6).

Both curves smoothed by running 3-point box-car averaging.

Figure 3 shows a comparison of the measured $\bar{j}_{e y}$ from run60 to the fit given by expression (6). It should be noted that there are no free parameters available to adjust the fit.

\section{Outflow Speed}

Expression (6) can be used to obtain an estimate of the electron outflow speed at a turning point; i.e., at the outflow boundary of the EDR. Assuming a steady state and ignoring the ion contribution to the current sheet strength,

$$
\frac{\Delta B_{x}}{d}=\frac{2 B_{i n}}{d}=\bar{j}_{e y}=n_{e_{X L}} \omega_{e c_{T P}}\left(\frac{L}{2}\right)=n_{e_{X L}} \frac{B_{o u t}}{m_{e}}\left(\frac{L}{2}\right)
$$

in which $B_{\text {in }}$ is the magnitude of $B_{x}$ at an inflow boundary of the EDR and $B_{\text {out }}$ is the magnitude of $B_{z}$ at an outflow boundary. Equating the second and last terms of (7) leads to

$$
B_{\text {in }}=\frac{1}{4} \frac{n_{e_{X L}}}{m_{e}} B_{\text {out }} L d=\frac{1}{4} B_{\text {out }} \hat{L} \hat{d}
$$

in which the carats indicate that the length and width are measured in units of the local electron inertial length. With $v_{\text {in }}$ for the magnitude of $v_{e z}$ at an inflow boundary to the EDR and with $v_{\text {out }}$ for the magnitude of $v_{e x}$ at an outflow boundary, it follows that $v_{i n} B_{\text {in }}=v_{\text {out }} B_{\text {out }}$ since $E_{y}$ is uniform over the 
EDR and the non-ideal parts of $E_{y}$ are, by definition, zero at the boundaries of the EDR. Multiplication of (8) by $v_{\text {in }}$ leads to $v_{\text {in }} B_{\text {in }}=v_{\text {out }} B_{\text {out }}=(1 / 4) v_{\text {in }} B_{\text {out }} \hat{L} \hat{d}$, or

$$
v_{\text {out }}=\frac{1}{4} v_{\text {in }} \hat{L} \hat{d}
$$

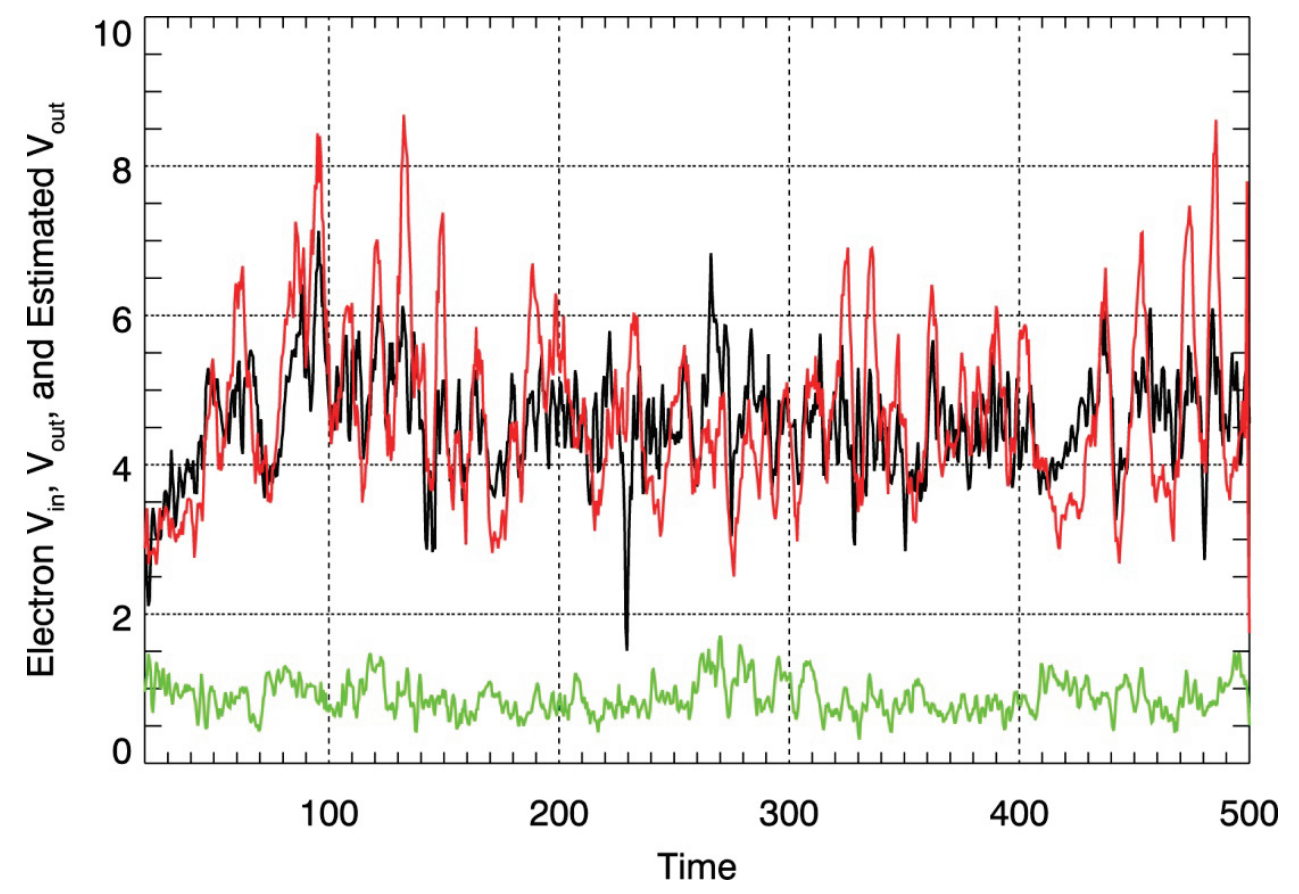

Figure 4: (color online) Green (light gray): Measured $v_{i n}$ (3-point running box-car averaged).

Black: Measured $v_{\text {out }}$ (3-point running box-car averaged).

Red (gray): $v_{\text {out }}$ fit given by (9) (9-point running box-car averaged).

Figure 4 shows the measured $v_{\text {in }}$ and $v_{\text {out }}$ along with the fit to $v_{\text {out }}$ given by (9). Again, there are no free parameters for adjustments. Notice the counter intuitive dependence on $\hat{d}$ rather than $1 / \hat{d}$.

\section{Reconnection Rate}

Inversion of (9) and division by $v_{\text {eAin }}$ leads to an expression for the reconnection rate

$$
\frac{v_{\text {in }}}{v_{\text {eAin }}}=4 \frac{v_{\text {out }}}{v_{\text {eAin }}} \frac{1}{\hat{L} \hat{d}}
$$

This expression can be simplified further. 


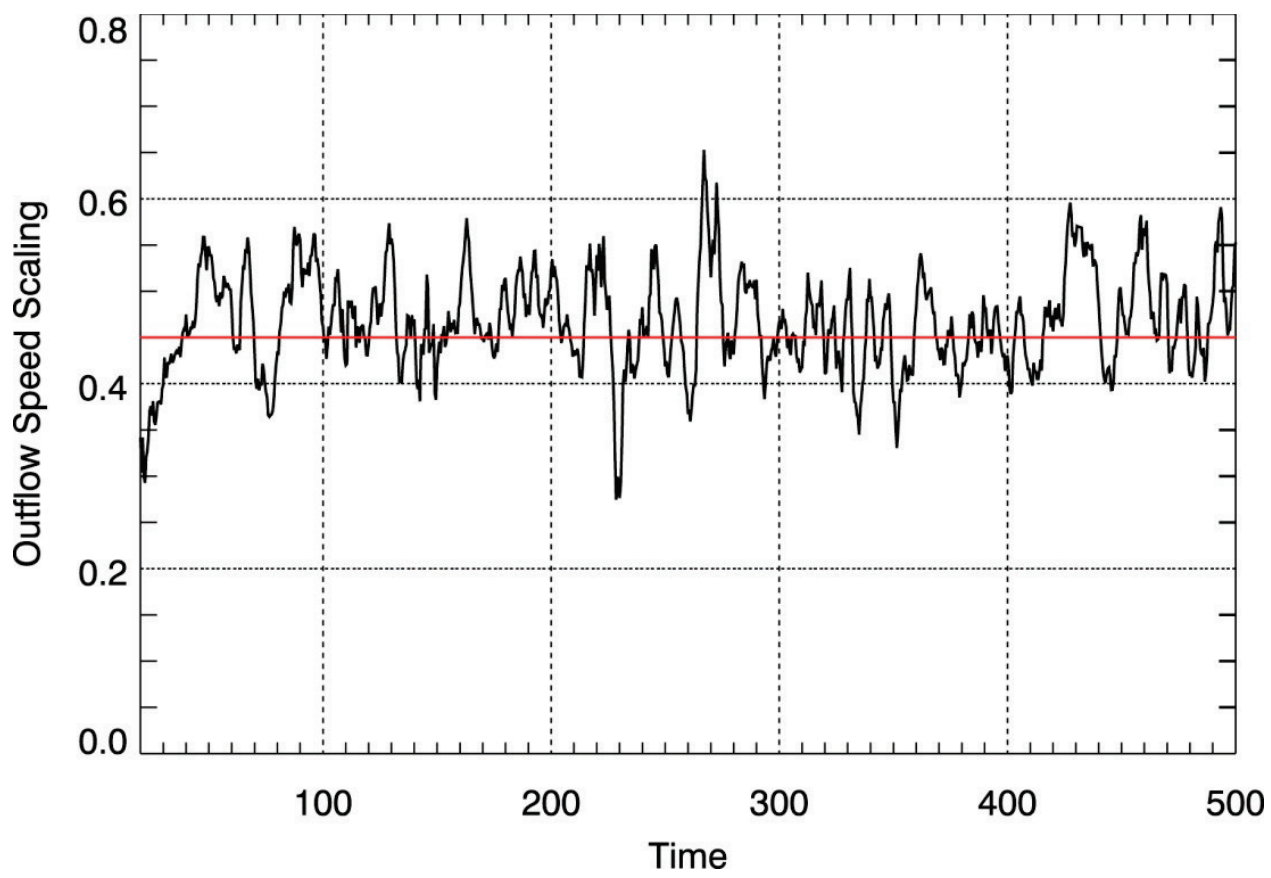

Figure 5: (color online) Black: Time evolution of ratio $v_{\text {out }} / v_{\text {eAin }}$. Red (gray): Time average of $v_{\text {out }} / v_{\text {eAin }}$ avoiding initial transients (Time $>100$ ). Smoothed by running 7-point box-car averaging.

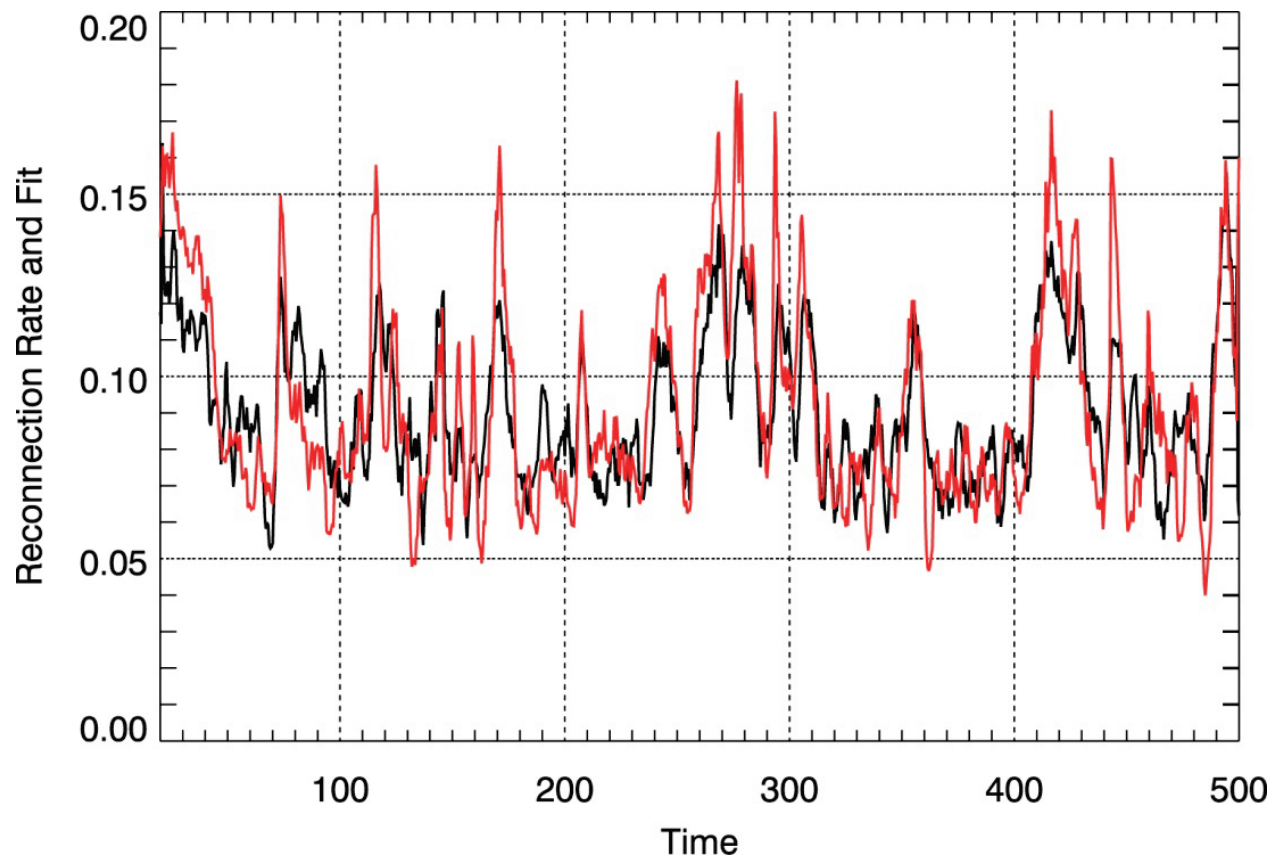

Figure 6: (color online) Black: Measured reconnection rate $v_{i n} / v_{\text {eAin }}$. Red (gray): Fit provided by reconnection rate expression (1). Both curves smoothed by running 7-point box-car averaging.

Figure 5 shows the evolution with time of the ratio $v_{\text {out }} / v_{\text {eAin }}$. It can be seen that, with the exception of an early transient, the ratio fluctuates about an almost constant value. It should be noted that, ignoring 
the small difference between inflow and outflow electron densities at the respective EDR boundaries, the existence of this almost constant value implies

$$
\frac{1}{2} m_{e} n_{\text {out }} v_{\text {out }}^{2} \simeq \frac{1}{4} \frac{B_{\text {in }}^{2}}{2}
$$

The electron outflow dynamic pressure at the EDR outflow boundary is approximately $1 / 4$ the inflow magnetic field pressure at the inflow boundary. Below, evidence that suggests this condition is generally true will be discussed.

Substitution of

$$
\alpha \equiv\left[\frac{v_{\text {out }}}{v_{\text {eAin }}}\right]_{\text {time averaged }}
$$

for the ratio $v_{\text {out }} / v_{\text {eAin }}$ in (10) leads to the reconnection rate expression (1). Figure 6 shows a comparison of the right to the left sides of (1) evaluated over the course of the run60 simulation with $\alpha=0.45$, the time-averaged value with the initial transient evident in Figure 5 excluded from the average. Again, it should be noted that there are no available free parameters for adjustments of this fit. The source of the overshoots in the fits to the peaks in the measured reconnection rate is under investigation.

\section{Out-of-Sample Performance}

The parameter $\alpha$ plays a prominent role in normalizing the reconnection rate expression (1). If $\alpha$ were to depend sensitively on specific details of a reconnection scenario, then the utility of (1) might be questioned. Some evidence suggesting a universal value for $\alpha$ has been found.

\begin{tabular}{c|cccc|} 
Simulation & $m_{i} / m_{e}$ & Driver Strength & Run Time $\left(\omega_{c i}^{-1}\right)$ & $\alpha$ \\
\hline run60 & 100 & 0.3 & 500 & 0.45 \\
run59 & 25 & 0.3 & 500 & 0.47 \\
run58 & 25 & undriven & 372 & 0.49 \\
run54 & 25 & 0.4 & 520 & 0.46 \\
run53d & 25 & 0.2 & 500 & 0.46
\end{tabular}

Table 1: (color online) $\alpha$ values for several simulations of various driving rates and two mass ratios

Table 1 shows the values for $\alpha$ that have been found for a group of simulations, including run60. In all cases $\alpha$ was obtained through the time average (12) with initial transient behavior excluded. Driver Strength is the value of the out-of-plane electric field imposed the inflow boundaries of a simulation following a short start-up interval during which the field strength was ramped up from zero. Comparison of run60 and run59 shows the effect of varying the mass ratio while holding the driver strength fixed. Simulations other than run60 show the effect of varying driver strength holding the mass ratio at 25 . For the undriven run58, the out-of-plane field at the inflow boundaries was allowed to float with a zero gradient imposed. Admittedly, Table 1 contains a small sample but it is difficult to ignore the suggestion 
that $\alpha$ represents a universal relationship between electron outflow dynamic pressure and inflow magnetic field pressure as expressed by (11).
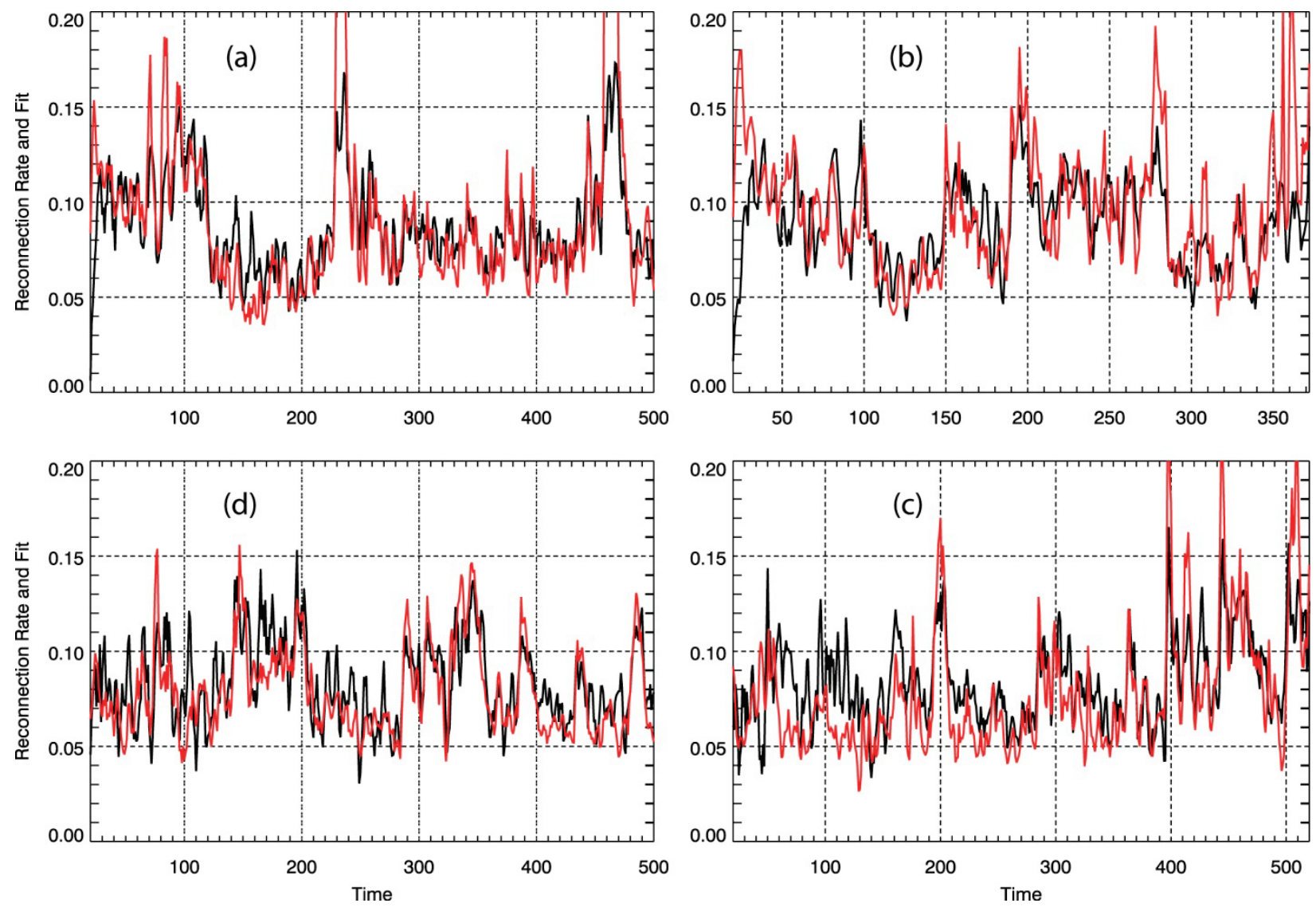

Figure 7: (color online) Black: Measured reconnection rate. Red (gray): Fit given by (1) for (a) run59, (b) run58, (c) run54, and (d) run53d using respective values for $\alpha$ from Table 1. All curves smoothed using running 3-point box-car averaging.

The performance of the reconnection rate expression (1) for run60 is shown in Figure 6. Figure 7 continues with examples of that performance for the remaining mass ratio 25 runs listed in Table 1. Again, there are no free parameters available and, again, there are occasional problems with excessive spikes in the fits. The source of these spikes is under investigation.

The degree to which the fits shown in Figure 7 succeed in reproducing the measured reconnection rates was unexpected. Two assumptions that were made in the derivation of (1) do not strictly apply. First, a cut in the $z$ variable through a mass ratio $25 \mathrm{EDR}$, as in Figure 1, generally yields a shape that is similar to that of the current distribution shown in panel (b) of Figure 1 (e.g., see Figure 6, Klimas et al. ${ }^{20}$ ). A central peak in the EDR, whose width coincides with that of the central peak in the current distribution, sits on top of a broader distribution that extends out to ion scales. As in the analysis of run60, the widths of these central peaks have been measured to fix the width $d$. Consequently, the non-ideal part of the out-of-plane electric field is not zero at the inflow boundary of the EDR, so defined, and the assumption leading to (9) that $E_{y}$ at the inflow boundary is given by $v_{i n} B_{i n}$ fails somewhat. Second, neglect of the 
ion current contribution to the strength of $B_{\text {in }}$ in (7) is less justified in the lower mass ratio simulations. The ion current contribution, averaged over the EDR width, can amount to as much as 15-20 percent of the electron contribution for the mass ratio 25 simulations; the ion contribution for the mass ration 100 run60 is typically around 5 percent. Despite these faults, the simple expression (1) yields surprisingly accurate fits to the measured reconnection rates for the mass ratio 25 as well as the mass ratio 100 simulations.

\section{EDR Width}

A straight forward derivation, based on fundamental conservation laws, shows that the reconnection electric field $E_{\text {rec }} \sim d / L^{17,18}$. A simplified portion of this derivation is given in this section. From (10), however, it also will be shown in this section that $E_{\text {rec }} \sim 1 / L d$. Resolution of this apparent contradiction imposes a new relationship that restricts the value of $\hat{d}$. The following applies strictly to run60 and approximately to the rest of the simulations listed in Table 1 for the reasons discussed in the preceding paragraph.

Starting with a box surrounding the EDR of length $L$ and width $d$, the length and width of the EDR, particle conservation for the electrons leads to

$$
\left\langle v_{\text {in }} n_{\text {in }}\right\rangle L=\left\langle v_{\text {out }} n_{\text {out }}\right\rangle d
$$

in which the brackets \langle\rangle indicate averages over the respective box edges. A detailed examination has shown that $\left\langle v_{\text {in }} n_{\text {in }}\right\rangle \simeq\left\langle v_{\text {in }}\right\rangle\left\langle n_{\text {in }}\right\rangle \simeq v_{\text {in }} n_{\text {in }}$ quite accurately. In addition, $\left\langle v_{\text {out }} n_{\text {out }}\right\rangle \simeq\left\langle v_{\text {out }}\right\rangle\left\langle n_{\text {out }}\right\rangle \simeq\left\langle v_{\text {out }}\right\rangle n_{\text {out }}$ but $\left\langle v_{\text {out }}\right\rangle \neq v_{\text {out }}$. On the outflow edges of the box, the outflow speed is peaked in the centers of the outflow jets such that $v_{\text {out }} \simeq 2\left\langle v_{\text {out }}\right\rangle$. From (13)

$$
v_{\text {in }} B_{\text {in }}=\left\langle v_{\text {out }}\right\rangle B_{\text {in }} \frac{d}{L}=E_{\text {rec }}
$$

in which $E_{r e c}$ is the reconnection field strength and $n_{\text {out }} / n_{\text {in }} \simeq 1$ has been assumed.

From (10) it can be seen that

$$
v_{\text {in }} B_{\text {in }}=4 v_{\text {out }} B_{\text {in }} \frac{1}{\hat{L} \hat{d}}=4 v_{\text {out }} B_{\text {in }} \frac{m_{e}}{n_{\text {eX }}} \frac{1}{L d}=E_{\text {rec }}
$$

Equating (14) and (15) leads to

$$
\hat{d}=2 \sqrt{\frac{v_{\text {out }}}{\left\langle v_{\text {out }}\right\rangle}}
$$




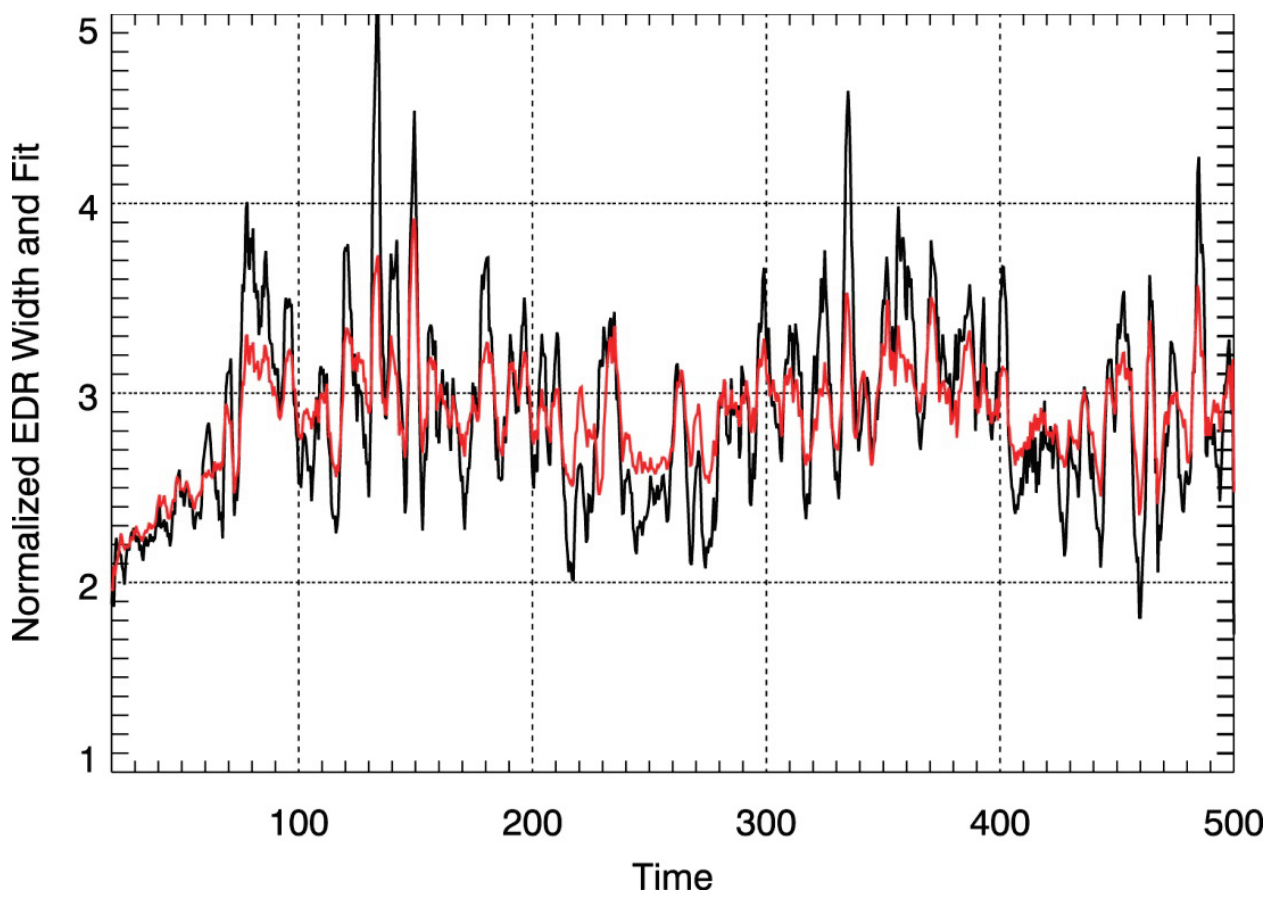

Figure 8: (color online) Black: Measured normalized EDR width. Red (gray): Fit given by (16). Both curves smoothed using running 7-point box-car averaging.

Figure 8 shows that the normalized EDR width does satisfy (16). Thus, the independent expressions (14) and (15) for the reconnection electric field strength are complementary and compatible, as are the independent methods used to obtain those expressions. Similar results have been obtained for all of the simulations listed in Table 1. Although, as in Figure 8, each of the simulations shows some variability in time, generally it has been found that $\hat{d} \simeq 3$, independent of the two mass ratios and various driving rates of the group.

\section{Discussion}

For symmetric, anti-parallel, collisionless reconnection a new expression for the reconnection rate has been derived in just a few simple steps from a physically intuitive starting point, the expression (6) for the electron current sheet strength. That expression shows that the average motion of a free electron at the x-line position in the current sheet should be viewed as the initial phase of a gyration about $B_{z}$ at a turning point. Surprisingly, the strength of the reconnection electric field does not play a direct role in fixing the current strength in (6).

In addition to the expression (6) for the electron current sheet strength, the derivation of the reconnection rate expression yields two interesting new results before the final expression is reached. The first of these is the expression (9) for the electron outflow speed at the EDR outflow boundary. A commonly employed concept of plasma conservation for plasma entering the EDR through the inflow boundary and then leaving through the outflow boundary suggests that, holding other parameters fixed, the outflow speed should depend inversely on the width $d$; as the width of the outflow boundary 
decreases outflow speed must increase to maintain a steady throughput. However, (9) states that the outflow speed is proportional to $d$ and Figure 4 confirms the accuracy of this expression for the outflow speed. This apparent contradiction, as well as the apparent contradictions indicated by the expressions (14) and (15) for the reconnection electric field strength, is removed by the restriction on $d$ given by (16). For the tightly coupled nonlinear reconnection system, once the system is specified, then the width $d$ is no longer free for adjustment. Plasma conservation applies but the commonly employed adjustability of $d$ does not.

The second new feature discovered on the way to the reconnection rate expression is the quasi-steady evolution over almost the entire run60 of the ratio $v_{\text {out }} / v_{\text {eAin }}$ as exhibited in Figure 5 , which leads to the approximate relationship between outgoing electron dynamic pressure and inflowing magnetic pressure given by (11). The final reconnection rate expression (1) is obtained by replacing this ratio by its time average $\alpha$. As exhibited in Table 1, $\alpha$ appears to be independent of mass ratio as well as driving rate for driven and undriven simulations. This unexpected result further increases the significance of (11) and also suggests an investigation of the invariance of $\alpha$ under additional changes in the reconnection scenario.

If the universality of $\alpha$ can be demonstrated, then all of the terms in the reconnection rate expression (1) except for the EDR length $L$ should be measurable in Earth's magnetotail by NASA's upcoming Magnetospheric Multiscale (MMS) mission (http://mms.space.swri.edu/index.html). Instead of $L$, it is possible that the equivalent turning length may be accessible from MMS measurements if three or four of the spacecraft pass through a single EDR sufficiently closely in time so that the dependence of the outflow field strength on displacement in the outflow directions can be modeled. If so, then the positions of the turning points may be modeled and the turning length estimated. Given that it will be possible, in this multi-spacecraft mission, to maintain the inter-spacecraft spacing at as small as $10 \mathrm{~km}$ the possibility of multiple spacecraft EDR passages is not out of the question.

In addition to observational verification of (1), if it is possible, further reconnection simulation studies are necessary. All of the results presented in this paper are noisier than one would wish; simulations with higher macro-particle densities should be carried out. The suggested convergence of the EDR and turning lengths with increasing mass ratio should be further tested. Verification would increase the likelihood of observational confirmation as discussed in the preceding paragraph. Confirmation of all of the results presented in this paper requires simulations using other available codes. Based on this experience, however, it is firmly believed that true open-boundary codes will be necessary.

Finally, the simplicity of the derivation discussed in this paper suggests that further extensions to the more realistic anti-symmetric and/or guide field reconnection models may be possible.

Acknowledgments: This research was supported by NASA's MMS IDS grant NCC5-494 (MOST). Invaluable discussions with Paul Cassak are greatly appreciated. 


\section{References}

${ }^{1}$ A. Nishida, Space Sci. Rev. 91 (3-4), 507-577 (2000).

${ }^{2}$ V. M. Vasyliunas, Space Sci. Rev. 158 (1), 91-118 (2011).

${ }^{3}$ R. A. Treumann, W. Baumjohann, arXiv:1401.5995v1 [physics.space-ph] (2014).

${ }^{4}$ V. M. Vasyliunas, Rev. Geophys. 13 (1), 303-336 (1975).

5. W. Dungey, La Recherche Spatiale 2 (15) (1989).

${ }^{6}$ M. Hesse, T. Neukirch, K. Schindler, M. Kuznetsova and S. Zenitani, Space Sci. Rev. 160 (1-4), 3-23 (2011).

${ }^{7}$ M. Hesse and D. Winske, J. Geophys. Res. 99 (A6), 11177-11192 (1994).

${ }^{8}$ R. Horiuchi and T. Sato, Phys. Plasmas 4 (2), 277-289 (1997).

${ }^{9}$ M. M. Kuznetsova, M. Hesse and D. Winske, J. Geophys. Res. 105 (A4), 7601-7616 (2000).

${ }^{10}$ J. Birn, J. F. Drake, M. A. Shay, B. N. Rogers, R. E. Denton, M. Hesse, M. Kuznetsova, Z. W. Ma, A. Bhattacharjee, A. Otto and P. L. Pritchett, J. Geophys. Res. 106 (A3), 3715-3719 (2001).

${ }^{11}$ H. Karimabadi, W. Daughton and J. Scudder, Geophys. Res. Lett. 34, L13104 (2007).

${ }^{12}$ K. Fujimoto and R. D. Sydora, Phys. Plasmas 16 (11) (2009).

${ }^{13}$ A. Divin, S. Markidis, G. Lapenta, V. S. Semenov, N. V. Erkaev and H. K. Biernat, Phys. Plasmas 17 (12) (2010).

${ }^{14}$ M. M. Kuznetsova, M. Hesse and D. Winske, J. Geophys. Res. 103 (A1), 199-213 (1998).

${ }^{15}$ M. Hesse, K. Schindler, J. Birn and M. Kuznetsova, Phys. Plasmas 6 (5), 1781-1795 (1999).

${ }^{16}$ M. M. Kuznetsova, M. Hesse and D. Winske, J. Geophys. Res. 106 (A3), 3799-3810 (2001).

${ }^{17}$ P. A. Cassak and M. A. Shay, Phys. Plasmas 14 (10) (2007).

${ }^{18}$ P. A. Cassak and M. A. Shay, Phys. Plasmas 16 (5) (2009).

${ }^{19}$ A. Klimas, M. Hesse and S. Zenitani, Phys. Plasmas 15, 082102 (2008).

${ }^{20}$ A. Klimas, M. Hesse, S. Zenitani and M. Kuznetsova, Phys. Plasmas 17, 112904 (2010).

${ }^{21}$ A. Klimas, M. Hesse and S. Zenitani, Phys. Plasmas 19, 042901 (2012). 